\title{
Targeting claudin-3 suppresses stem cell-like phenotype in nonsquamous non-small-cell lung carcinoma
}

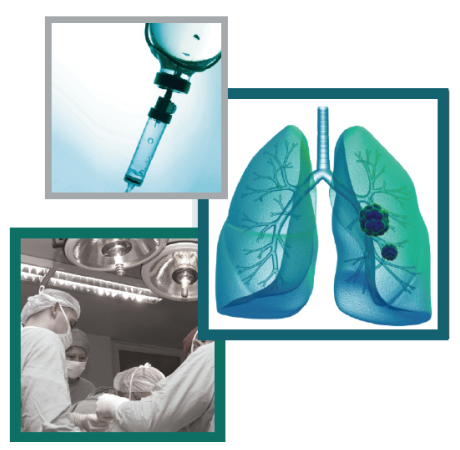

\author{
Lin Ma*,‡,1,2,3, Wu Yin ${ }^{\ddagger} 3$, Heliang Ma ${ }^{\ddagger} 4$, Ihab Elshoura ${ }^{3}$ \& Lan Wang ${ }^{5}$ \\ ${ }^{1}$ School of Materials Science \& Engineering, South China University of Technology, Guangzhou 510640, China \\ ${ }^{2}$ R\&D Center, Guangzhou Ribobio Co., Ltd, Guangzhou 510663, China \\ ${ }^{3}$ State Key Laboratory of Pharmaceutical Biotechnology, School of Life Sciences, Nanjing University, Nanjing 210046, China \\ ${ }^{4}$ Department of Radiology, Jinan Central Hospital, Jinan, Shandong 250013, China \\ ${ }^{5}$ Department of Respiratory Medicine, The Affiliated Jiangyin Hospital of Southeast University, Jiangyin 214400, China \\ *Author for correspondence: Xeno.ma@163.com \\ $\ddagger$ Authors contributed equally
}

\begin{abstract}
Aim: To determine the role of claudin-3 in cancer stemness in nonsquamous non-small-cell lung carcinoma (NSCLC). Materials \& methods: In vitro/vivo extreme limiting dilution analysis and the side population assay were used to investigate the role of claudin-3 in regulating cancer stemness in nonsquamous NSCLC. Results \& conclusion: Claudin-3 depletion decreased the formation rates of spheres and tumors and increased cisplatin sensitivity. Claudin-3 was also identified as one downstream target of estrogen receptor$\alpha$ in regulating cancer stemness. Moreover, targeting CLDN-3 transcription by small molecules including withaferin A, estradiol and fulvestrant suppressed cancer stemness and reversed chemoresistance. These results demonstrated claudin-3 is one positive regulator of cancer stemness in nonsuqamous NSCLC.
\end{abstract}

First draft submitted: 8 May 2018; Accepted for publication: 8 November 2018; Published online: 26 February 2019

Keywords: cancer stemness $\bullet$ cisplatin $\bullet$ claudin-3 $\bullet$ CSCs $\bullet$ ELDA $\bullet$ estrogen receptor- $\alpha \bullet$ nonsquamous NSCLC $\bullet$ side population • tight junction • tumorigenesis

Lung cancer represents one global health problem and still remains the first leading course of cancer-related deaths worldwide [1]. Non-small-cell lung carcinoma (NSCLC), a heterogeneous class of tumors representing approximately $85 \%$ of all new lung cancer diagnoses, contains squamous cell lung carcinoma (SCC) and nonsquamous NSCLC of the lung including large-cell lung carcinoma (LCLC) and adenocarcinoma (AC) [2]. Notably, nonsquamous NSCLC is the most common malignant type both in male and female nonsmokers and usually insensitive to chemotherapy and/or radiation. At present, surgery still remains the treatment of choice if patients are diagnosed with nonsquamous NSCLC at an early stage and platinum-based chemotherapy remains the standard of care for most patients affected by advanced nonsquamous NSCLC [3]. However, with the advent of metastasis and tumor relapse, the effects of traditional therapies in more advanced nonsquamous NSCLC cases are frequently temporary, and after months of treatment the disease progresses to its lethal stage with a short survival period [4].

Recent studies revealed that cancer stem-like cells (CSCs), which are enriched after chemotherapy, may account for therapy failure. In typically heterogeneous tumors, CSCs represent a small group of cells which possess tumorinitiating, self-renewal properties and give rise to nontumorigenic progeny. More and more data demonstrated that CSCs play a pivotal role in the tumor relapse, metastasis and therapeutic refractoriness [5,6]. Moreover, implementation of treatments against CSCs suppresses chemoresistance and increases the effectiveness of conventional therapy across a spectrum of malignancies [7]. Thus, CSC-focused therapy seems destined to be the core of effective anticancer approaches.

Claudin proteins are crucial components of cellular tight junctions where they establish the paracellular barrier to control the flow of molecules in the intercellular space between epithelial cells [8]. To date, the claudin family in mammals is composed of 24 members with molecular weights ranging from 22 to $27 \mathrm{kDa}$, all of which are thought to vary in expression depending on location and cell type [9]. Notably, the link between altered tight

Future Medicine 
junctions and epithelial tumor development has been confirmed by earlier studies placing tight junctions in the spotlight of cancer study, and this research area has become very promising as a result of the frequent existence of altered claudin expression in cancer [10]. Among these claudin members, overexpression of claudin-3, claudin- 4 and downregulation of claudin-7 were demonstrated in lung malignancies [11-14]. Although their dysregulation in malignancies suggests that claudin proteins play a role in tumorigenesis [15-18], the exact underlying mechanism remains unclear. As a member of the claudin family, claudin-3, also named Clostridium perfringens enterotoxin receptor 2, is encoded by the $C L D N-3$ gene and located on chromosome 7 . As an epigenetically silenced tumor suppressor gene in hepatocellular carcinoma, claudin-3 has been reported to inhibit cancer aggressiveness via WNT-EMT signaling, and loss of claudin-3 leads to colon cancer malignancy by hyperactivating Wnt/ $\beta$-catenin signaling $[19,20]$. However, increasing lines of evidence suggest that claudin-3 could play an oncogenic role in some malignancies [21,22]. Although several other members of the claudin family, including claudin-1 and claudin-2, function as tumor facilitators and promote tumorigenesis [23,24], the precise role for claudin-3 in cancer stemness still remains to be investigated.

Side-population (SP) cells are a group of cells which cannot be stained with Hoechst 33342, as opposed to those cells treated with the pump inhibitor verapamil. It has been reported that the SP cells in human lung cancer cell line NCI-H460 are enriched in stem-like cancer cells [25], and thus we defined CSCs in NSCLC on the basis of the SP phenotype. As the golden scale to evaluate the tumor stemness, the in vitro or in vivo limited dilution assay (ELDA) has also been used to evaluate the efficiency of tumor-sphere formation or tumor generation in nude mice. By these two methods, we identified claudin-3 as a positive regulator of cancer stemness and CSCs-mediated chemoresistance in nonsquamous NSCLC. Using a CLDN-3 transcription-based drug screening assay, small molecules including estradiol, withaferin A (WA) and fulvestrant were all found to downregulate the transcriptional activity of claudin-3 and suppress cancer stemness in nonsquamous NSCLC. Furthermore, antagonizing estrogen receptor- $\alpha$, a newly developed positive prognostic indicator of NSCLC, could suppresses cancer stemness via downregulation of claudin-3, indicating that claudin-3 could function downstream of estrogen receptor- $\alpha$ (ER- $\alpha)$ signaling in mediating cancer stemness in nonsquamous NSCLC. In sum, targeting claudin-3 may provide an innovative strategy worth considering nonsquamous NSCLC therapy.

\section{Materials \& methods}

Cell culture

Five nonsquamous NSCLC cell lines (H460, H1792, H157, H292 and A549) were purchased from American Type Culture Collection (ATCC; VA, USA). The A549 and H292 were maintained in DMEM medium containing $10 \%$ of fetal bovine serum (FBS) and $50 \mathrm{U} / \mathrm{ml}$ of penicillin/streptomycin. The remaining cell lines including H460, H1792 and H157 were cultured using RPMI1640 medium containing 10\% of FBS and 50 U/ml penicillin/streptomycin. Briefly, cells were cultured using a $6 \mathrm{~cm}$ dish with the split-ratio 1:5 every 2 days. Cells in $\log$-phase were harvested using trypsin-EDTA solution. The cell cultures were incubated at $37^{\circ} \mathrm{C}$ with a humidified atmosphere containing $5 \%$ of $\mathrm{CO}_{2}$.

\section{siRNA transfections}

Cells were transfected with pools of scrambled or target gene-specific siRNAs (100 nM) using Lipofectamine 2000 according to the manufacturer's instructions. The sequences of designed siRNAs targeting claudin- 3 were as follows (sense): siCLDN-3-1 5'-GCUACGACCGCAAGGACUA-3', siCLDN-3-2 5'-AGAUGCAGUGCAAGGUGUA$3^{\prime}$. The siRNA sequence for $E R-\alpha$ was (sense): GCUACUGUGCAGUGUGCAA.

\section{Lenivirus-mediated knockdown}

The H460 stable claudin-3 knockdown cells line was created by lentiviral transduction of a pLentilox3.7 vector containing a specific construct (CLDN-3 shRNA sense 5'-GCTACGACCGCAAGGACTA-3'). Package of recombinant lentivirus was performed by transfection of 293T cells. Briefly, shRNA expression vector pLentilox3.7shCLDN-3 or pLentilox3.7-null was cotransfected with package vectors pCMV $\Delta 8.9$ and pCMV-VSVG into 293 T cells using Lipofectamine $2000^{\circledR}$ (Invitrogen, Paisley, Scotland). Lentiviral particles in the culture media were harvested at $72 \mathrm{~h}$ after transfection and filtered through a $0.46 \mathrm{um}$ low protein binding polysulfonic filter (Millipore, MA, USA). Then lentiviral particles were enriched into the CSCs media, DMEM/F12 serum-free medium supplemented with $20 \mathrm{ng} / \mathrm{ml} \mathrm{EGF} \mathrm{(BD} \mathrm{Pharmingen,} \mathrm{CA,} \mathrm{USA),} 20 \mathrm{ng} / \mathrm{ml}$ of bFGF (BD Pharmingen), $0.4 \%$ of BSA (Sigma-Aldrich, MO, USA), 2\% of B27 (BD Pharmingen) and 1\% of methyl cellulose (Sigma-Aldrich) after 
ultracentrifugation. Cells were transfected with $1 \times 10^{6}$ of TU/ml lentiviral particles and sorted using BD Aria software after $72 \mathrm{~h}$ of transfection. To enhance the infection efficacy of lentiviral particles, $6 \mu \mathrm{g} / \mathrm{ml}$ of polybrene was added to the cells. For tumorsphere transfection, doubled lentivirus transfection procedure was performed. Briefly, 7-day lung tumorspheres were transfected with $10 \mathrm{ul}$ of $1 \times 10^{6} \mathrm{TU} / \mathrm{ml}$ lentivirus. The lentivirus-containing medium was replaced and $30 \mathrm{ul}$ of $1 \times 10^{6} \mathrm{TU} / \mathrm{ml}$ lentivirus was added to the cells in 2 days. The medium was also changed after $24 \mathrm{~h}$ and tumorspheres were cultured for another 7 days. Then tumorspheres were treated with cisplatin for 12 or $30 \mathrm{~h}$ and the cellular uptake of cisplatin or apoptosis was detected.

\section{Antibodies}

Rabbit polyclonal antibody against claudin-3 (catalog number NB100-91711) was purchased from Novus Biological USA (CO, USA). Mouse monoclonal antibody against ER- $\alpha$ (catalog number GTX70171) and rabbit polyclonal antibodies against CD133 (catalog number GTX12295), ALDH1A1 (catalog number GTX123974) and ABCG2 (catalog number GTX100437) were purchased from GeneTex (CA, USA). Mouse monoclonal anti-GAPDH IgG (catalog number sc-47724) and HRP-conjugated secondary IgGs (catalog number sc-2054 and sc-2969) were purchased from Santa Cruz Biotechnology (Santa Cruz, CA, USA).

\section{Sorting \& analysis of SP cells}

Cells were trypsinized and resuspended at $1 \times 10^{6}$ cells $/ \mathrm{ml}$ in DMEM or RPMI1640 medium with $2 \%$ of FBS containing $5 \mu \mathrm{g} / \mathrm{ml}$ of Hoechst 33342 at $37^{\circ} \mathrm{C}$ for $90 \mathrm{~min}$ with or without $100 \mu \mathrm{M}$ verapamil (Sigma) to inhibit $\mathrm{ABC}$ transporters. Then cells were incubated on ice for $10 \mathrm{~min}$ and washed with ice-cold PBS before flow cytometric sorting and analysis. Propidium iodide (PI) was used to distinguish live and dead cells, and the HoechstRed/HoechstBlue negative and PI negative population represents the group of SP. The percentages of SP were detected with BD Aria software and analyzed using FlowJo 7.6.1 software.

\section{Lung-tumorspheres formation assay}

Cells were seeded in ultra-low cluster plates (Corning Inc., NY, USA) and cultured in DMEM/F12 serum-free medium supplemented with $20 \mathrm{ng} / \mathrm{ml}$ EGF, $20 \mathrm{ng} / \mathrm{ml}$ bFGF, $0.4 \%$ BSA and 2\% B27 as well as $1 \%$ of methyl cellulose (Sigma-Aldrich). For ELDA in vitro, cells at different densities (1, 10, 100 and 1000/well) were cultured, 12 wells per cell density, in stem cell medium in 96-well plates $(200 \mu \mathrm{l} /$ well) for 1 to 2 weeks. The numbers of wells with at least one tumorsphere (diameter $>50 \mu \mathrm{m}$ ) were counted in a blinded manner. Frequencies of sphere-initiating cells were calculated by ELDA online program [26].

\section{Tumorigenicity in vivo}

Different numbers of cells were resuspended in RPMI1640 medium without FBS and subsequently injected (200 $\mu$ l for each injection) subcutaneously into the flanks of male BALB/c nude mice (Model Animal Research Center of Nanjing) at 3 weeks of age, which were housed in a specific pathogen-free facility. The visible xenograft tumors were counted manually after 18 days of injection and the tumor formation rate was calculated using ELDA online program. Procedures were carried out according to the National Institutes of Health Guide for the Care and Use of Laboratory Animals (NIH publication number 80-23) revised 1996.

\section{Apoptosis assay}

To detect sensitivity of $\mathrm{H} 460$ cells or tumorspheres, cells/tumorspheres were treated with $10 \mu \mathrm{g} / \mathrm{ml}$ of cisplatin for $30 \mathrm{~h}$ followed by apoptosis detection using the Annexin V-FITC/PI double-staining method. Briefly, tumorspheres were collected and trypsinized into single cells which were then washed and resuspended in binding buffer followed by AnnexinV-FITC/PI staining. The frequencies of apoptosis were detected using FACS Calibur software and data were analyzed using FlowJo 7.6.1 software.

\section{Cisplatin uptake}

Cells or tumorspheres were treated with $10 \mu \mathrm{g} / \mathrm{ml}$ of cisplatin for $12 \mathrm{~h}$, and harvested cells were dispersed in water and nitrolyzed by adding $\mathrm{HNO}_{3}{ }^{+}$into the solution and heating at $95^{\circ} \mathrm{C}$ for $2 \mathrm{~h}$. The solution was then treated with $\mathrm{H}_{2} \mathrm{O}_{2}$ at $95^{\circ} \mathrm{C}$ for $2 \mathrm{~h}$ and with $\mathrm{HCl}$ at $37^{\circ} \mathrm{C}$ for $0.5 \mathrm{~h}$. The content of Pt was determined by inductively coupled plasma mass spectrometry after dilution to $1 \mathrm{ml}$. The average of three independent results was taken as the final value for Pt. 


\section{Western blot}

Cells were washed with 1x PBS and lysed on an ice with RIPA buffer supplemented with a protease inhibitor cocktail (P8340; Sigma-Aldrich). Lysates were subjected to SDS/PAGE followed by blotting with the indicated antibodies. Signal detection was achieved using Clarity Western ECL substrate (Bio-Rad).

\section{RNA extraction \& RT-PCR}

Total RNAs were isolated with TRIzol reagent (Invitrogen) following the manufacturer's instructions. The RT-PCR was performed in $20 \mu \mathrm{l}$ of reaction mixture. The PCR products were resolved on $1.2 \%$ of agarose gels and stained with GelRed. Glyceraldehyde 3-phosphate dehydrogenase was also detected as a loading control.

\section{Statistics}

Results are expressed as mean \pm standard deviation. Student's t-test or two-way ANOVA analysis followed by Bonferroni post hoc test were used to determine the statistical significance of the differences between experimental groups; p-values less than 0.05 were considered statistically significant. All data were processed with GraphPad Prism 5.0 software.

\section{Results}

Claudin-3 is highly expressed in CSCs \& positively correlated with cancer stemness in nonsquamous NSCLC

By analyzing $C L D N-3$ expression in lung adenocarcinoma and normal lung tissues according to the TCGA and GTEx data using the GEPIA online program developed by Z Tang, C Li and B Kang of Zhang Lab in Peking University [27], $C L D N-3$ was found to be highly expressed in lung adenocarcinoma (Figure 1A). We next utilized the GDS3837 (provided by R Kuner) and GDS3627 (provided by TP Lu) gene expression profile from the curated datasets in the Gene Expression Omnibus repository. Analyses of differently expressed genes between paired tumor sections and adjacent normal lung tissue specimens obtained from nonsmoking female NSCLC patients represent an abundantly expressed claudin-3 in lung malignant tissues (Figure 1B). This observation suggests an important role of claudin-3 in nonsquamous NSCLC. According to the GSE54712 [28] gene expression profile provided by BD Lopez-Ayllon [29], $C L D N-3$ was found to be overexpressed in CSCs isolated from $\mathrm{H} 460$, which was subsequently downregulated in redifferentiated cells under serum-containing culturing condition (Figure 2A). Thus, claudin-3 might be a potential indicator of cancer stemness in nonsquamous NSCLC. To test this hypothesis, we next examined claudin-3 expression in CSCs. By Hoechst33342-based SP sorting followed by western blot and RT-PCR assays, higher levels of claudin-3 protein and mRNA were both detected in H460 SP with higher levels of CSC markers including CD133, ABCG2 and ALDH1A1 compared with those in unfractionated H460 (Figures 2B \& C). Meanwhile, abundant expression of claudin-3 was also observed in H460 tumorspheres compared with that in the H460 parental cell line (Figure 2D). To further confirm the association between claudin-3 expression and the CSCs phenotype, percentages of SP were detected in other three NSCC cell lines including A549, H157 and $\mathrm{H} 292$, with a proportion of 0.6-3.5\%. Notably, the percentage of SP in cells with higher claudin-3 protein level was significantly higher than that in cells with less or no expression of claudin-3, indicating claudin-3 positively correlated with cancer stemness in nonsquamous NSCLC (Figures 2E \& F).

\section{Claudin-3 positively regulates cancer stemness \& CSCs-mediated resistance to cisplatin in nonsquamous NSCLC}

To investigate the role of claudin-3 in the maintenance of CSCs phenotype, RNAi-mediated claudin-3 knockdown assay was performed, revealing that depletion of claudin-3 decreased the SP percentages in both H460 and H1792 (Figures 3A \& B). To validate the function of claudin-3 in regulating cancer stemness in nonsquamous NSCLC, the tumorsphere formation assay and ELDA analysis were performed using stable transfected shCLDN-3 H460 cell line (vector as the control). Based on the observation, depletion of claudin-3 downregulated the capacity of the lung-tumorsphere formation (Figures 3C \& D). Moreover, knockdown of claudin-3 decreased the tumorsphere formation rate in vitro and the tumor formation rate in vivo (Figures $3 \mathrm{E} \& \mathrm{~F}$ ). Thus, claudin-3 was a positive regulator of cancer stemness in nonsquamous NSCLC.

In view of the roles of CSCs in therapeutic refractoriness of tumors, it was therefore necessary to investigate the involvement of claudin-3 in CSC-mediated chemoresistance. By examining cisplatin sensitivities and the intracellular cisplatin level, downregulated cisplatin-induced apoptosis and decreased cellular uptake of cisplatin 
(A)

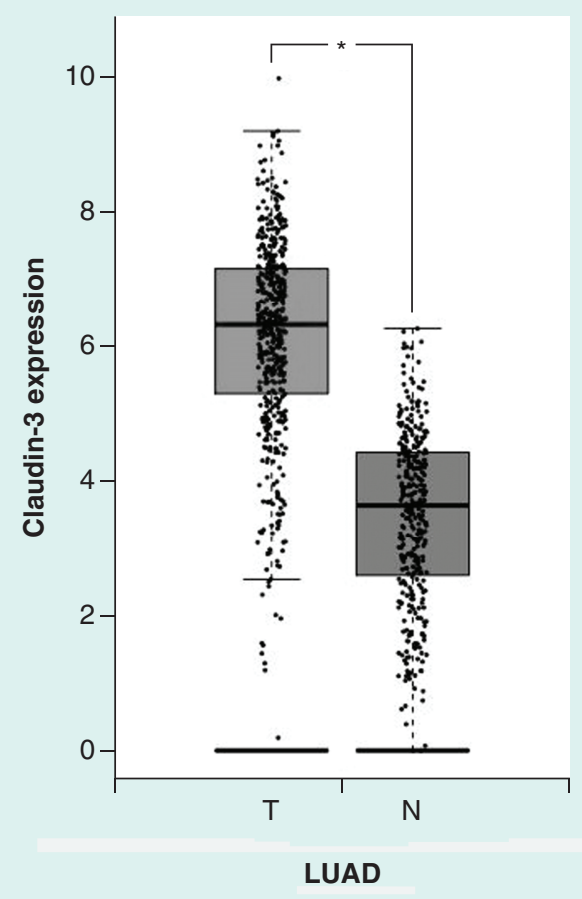

(B)

GDS3837:

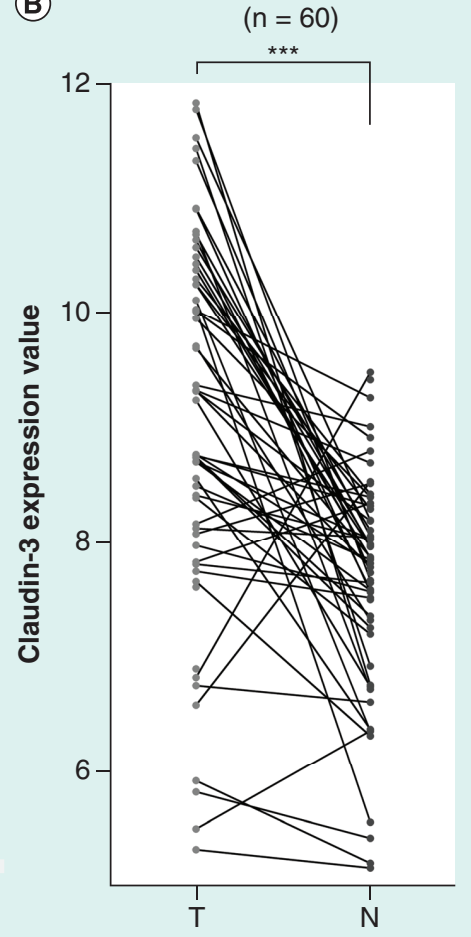

Figure 1. Claudin-3 is overexpressed in nonsquamous non-small-cell lung carcinoma. (A) Detection of CLDN-3 expression in LUAD $(n=483)$ and normal lung tissues $(n=347)$ according to The Cancer Genome Atlas

(TCGA) database and The Genotype-Tissue Expression (GTEx) database. (B) CLDN-3 expression analysis of paired tumor and adjacent normal lung tissue specimens obtained from nonsmoking female non-small-cell lung carcinoma patients in Taiwan obtained from Gene Expression Omnibus database (GDS3837)

(www.ncbi.nlm.nih.gov/sites/GDSbrowser?acc=GDS3837). ${ }^{*} p<0.05, * * * p<0.001$

$\mathrm{N}$ : Normal samples; T: Tumors.

were both observed in H460 tumorspheres compared with the parental H460 (Figures 4A \& B). To further confirm the role of claudin-3 in mediating cisplatin resistance, lentivirus particle-mediated claudin-3 knockdown assays were performed and the results showed that depletion of claudin-3 in tumorspheres reversed chemoresistance and increased intracellular cisplatin content (Figures 4C \& D). Meanwhile, claudin-3 knockdown in H460 tumorspheres by lentivirus transfection resulted in cleavage of caspase- 9 and downstream PARP after treatment of cisplatin (Figure 4E). These observations indicate that targeting claudin-3 could provide a promising strategy to overcome chemoresistance mediated by CSCs in nonsquamous NSCLC.

\section{Screening of alaudin-3 inhibitors to suppress cancer stemness in nonsquamous NSCLC}

In an effort to select drugs targeting claudin-3 to suppress cancer stemness and its associated chemoresistance in NSCC, a claudin-3 transcription-based dual luciferase reporter system was established and quantitative claudin-3 promoter activity profiles from H460 cells treated with ten drugs were obtained. Significantly, estradiol, WA and fulvestrant could all downregulate the transcription activity of claudin-3 as well as its protein levels and decrease the percentage of SP (Figures 5A-C). Among these drugs, WA has shown the strongest inhibitory effect on claudin-3 transcription and the maintenance of the SP group. Thus, we next confirm the role of WA in regulating cancer stemness via in vitro tumorsphere assay and in vivo tumor formation assay. In consistent with the above observations, WA significantly downregulated the formation capacity of $\mathrm{H} 460$ tumorspheres and decreased the sphere formation rate, which required the role of claudin-3 (Figures 5D-F). Moreover, WA markedly increased sensitivity of H460 tumorspheres toward cisplatin, which was compromised in the absence of claudin-3 (Figure 5G). Thus, claudin-3 has been proved to be a potential target for NSCC therapy against CSCs. 


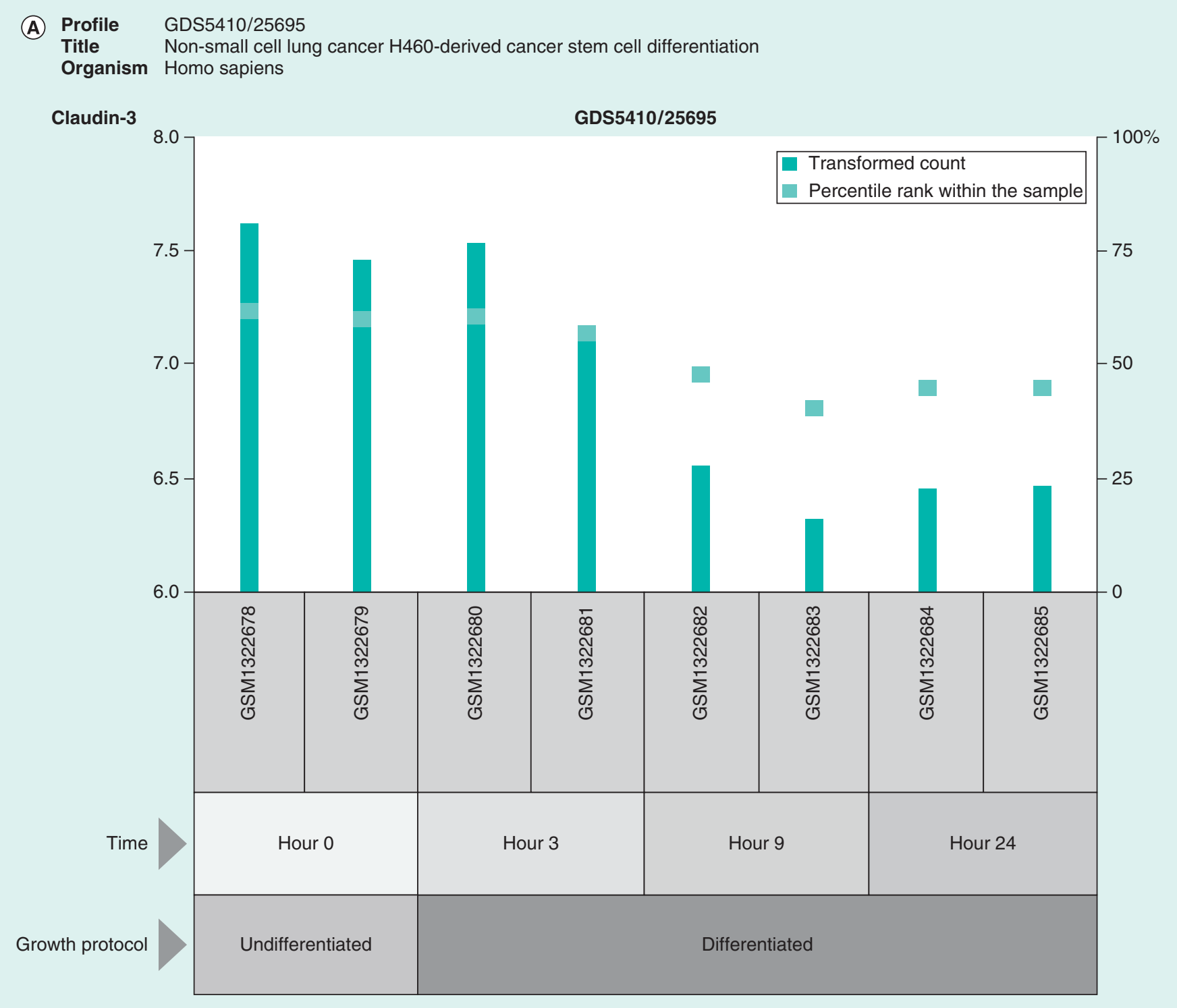

Figure 2. Claudin-3 expression is associated with cancer stemness in nonsquamous non-small-cell lung carcinoma. (A) CLDN-3 expression profile from Gene Expression Omnibus database (GDS5410) in which CSCs were isolated from $\mathrm{H} 460$ and allowed to redifferentiate under adherent conditions in serum-containing media for up to $24 \mathrm{~h}$. (B) Sorting of SP from H460 cells using BD Aria. Verapamil was used as the negative control for SP analysis. (C) Expression of claudin-3 as well as CSCs markers in H460 and H460 SP. (D) Detection of claudin-3 protein levels between parental H460 cells and H460 tumorspheres. (E) Detection of claudin-3 protein levels among nonsquamous NSCLC cell lines including A549, H157 and H292. (F) Detection of SP percentage among various non-squamous NSCLC cell lines including A549, $\mathrm{H} 157$ and H292. Data of SP analysis from triplicates are presented as the mean \pm standard deviation; ${ }^{*} p<0.05 ; * * p<0.01 ; * * * p<0.001$. CSC: Cancer stem-like cell; NSCLC: Non-small-cell lung carcinoma; SP: Side-population.

Antagonizing estrogen receptor- $\alpha$ inhibits cancer stemness by suppressing downstream claudin-3 in nonsquamous NSCLC

We noticed that the three identified drugs targeting claudin-3, including estradiol, WA, and fulvestrant, were inhibitors of ER- $\alpha$, demonstrated by previous reports [30-32]. By coincidence, altered transcriptome profiles between nonsquamous NSCLC and SCC showed that both ER- $\alpha$ and claudin-3 were preferentially expressed in nonsquamous NSCLC rather than in SCC (Figure 6A). Notably, claudin-3 was dramatically downregulated with decreased level of CSCs markers in ER- $\alpha$-depleted MCF7 cell lines (Figure 6B). To confirm the relationship between claudin-3 and ER- $\alpha$, we performed knockdown assays and observed that ER- $\alpha$ depletion dramatically 
(B)
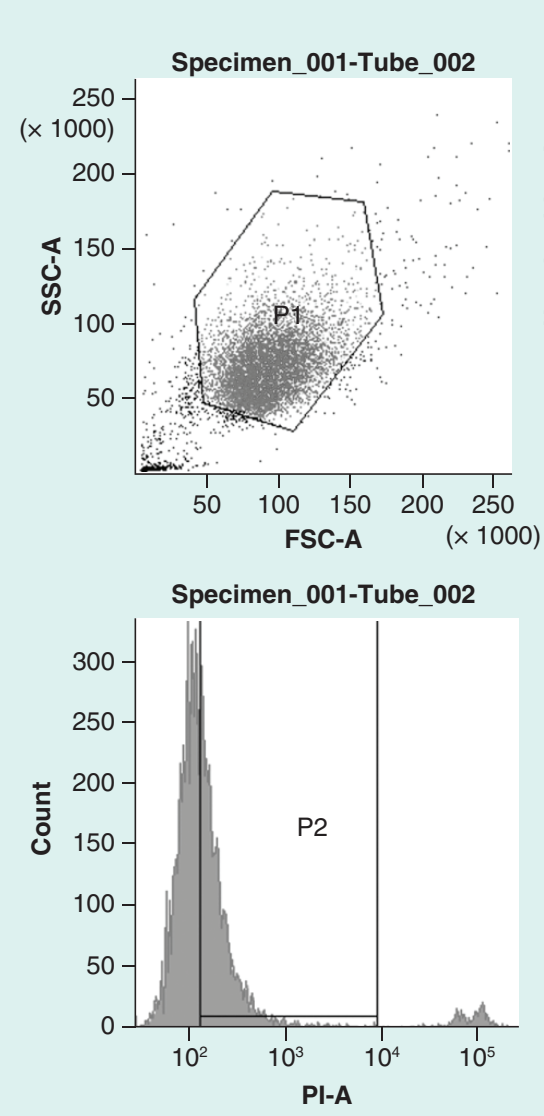
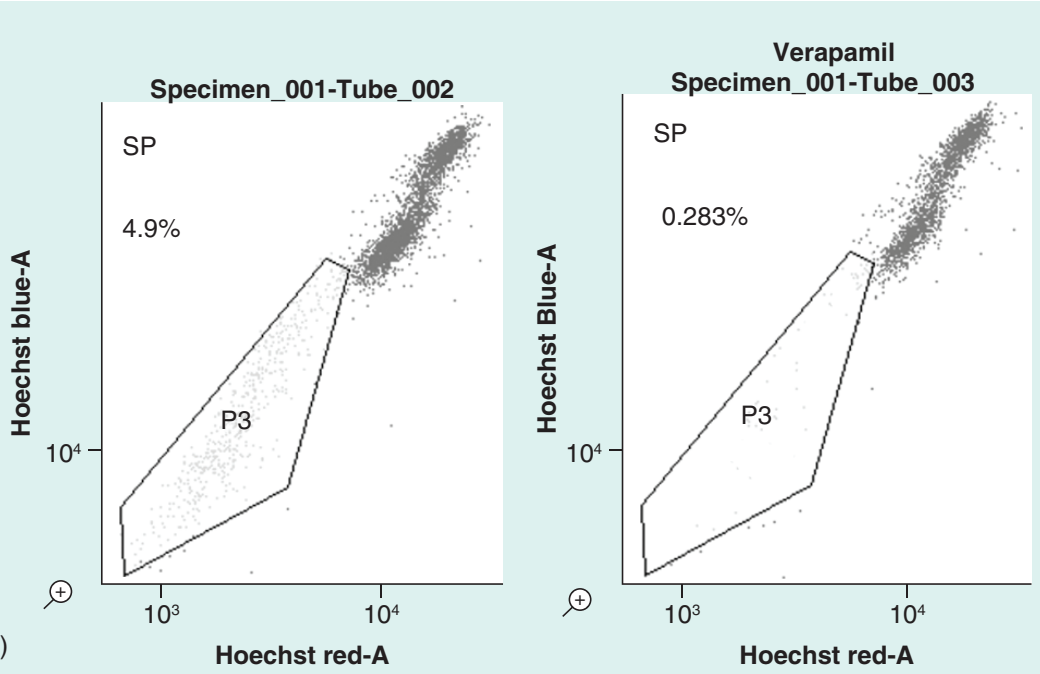

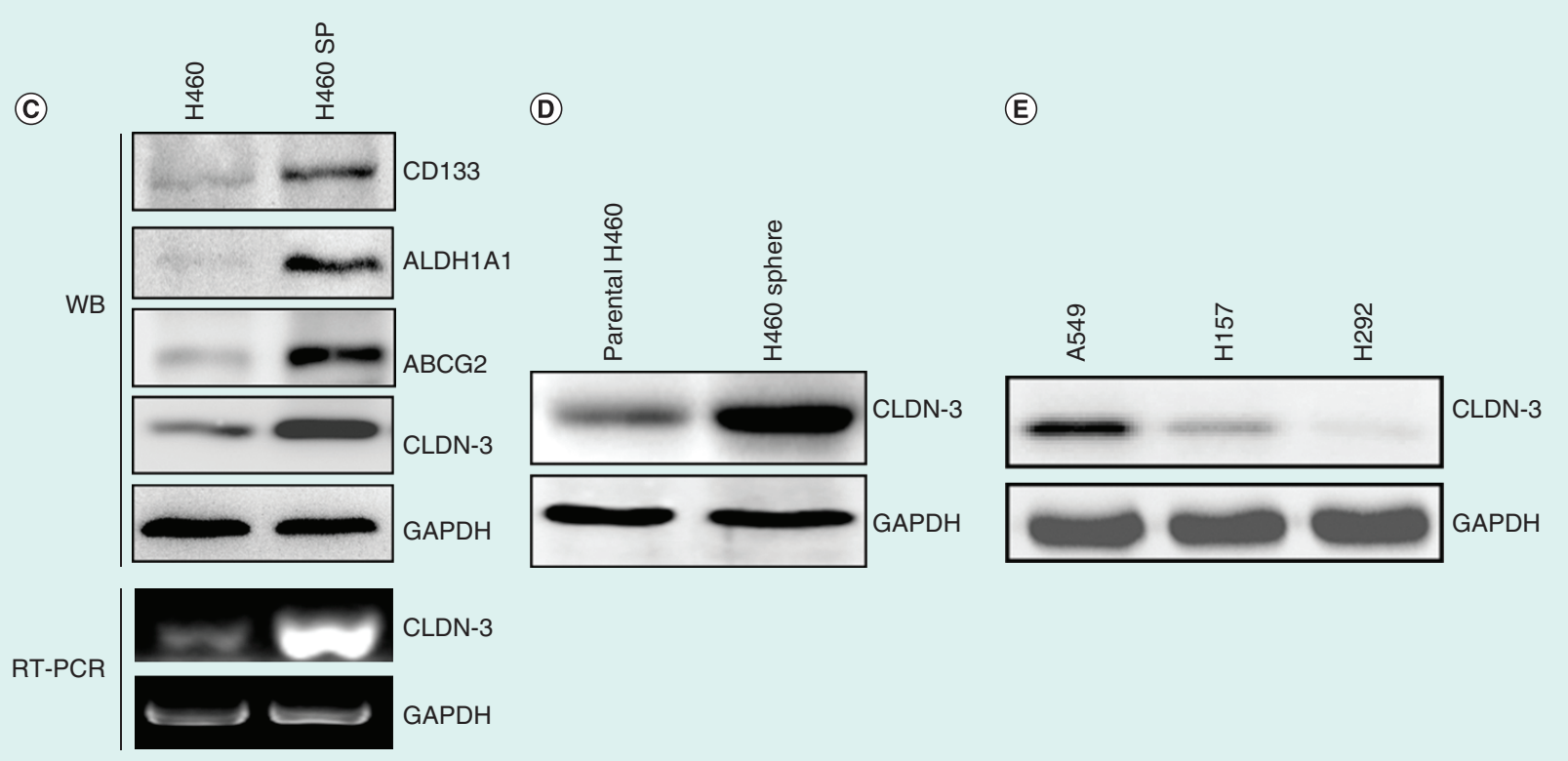

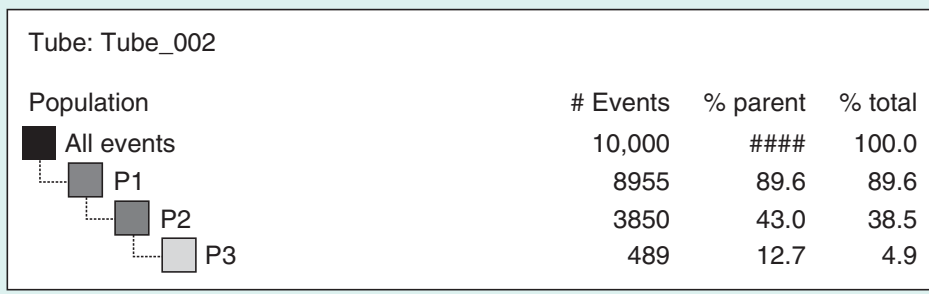

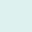



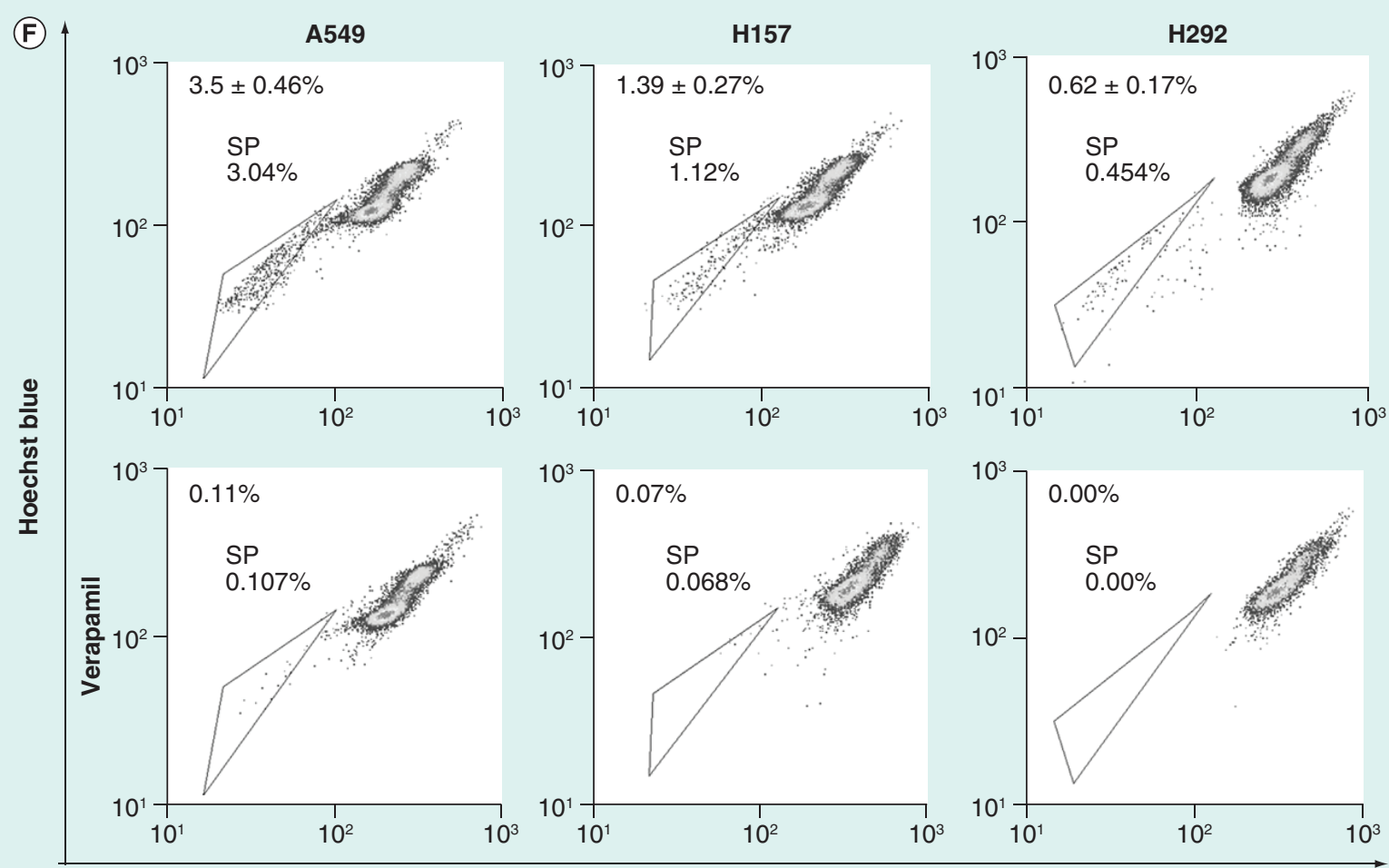

Hoechst red

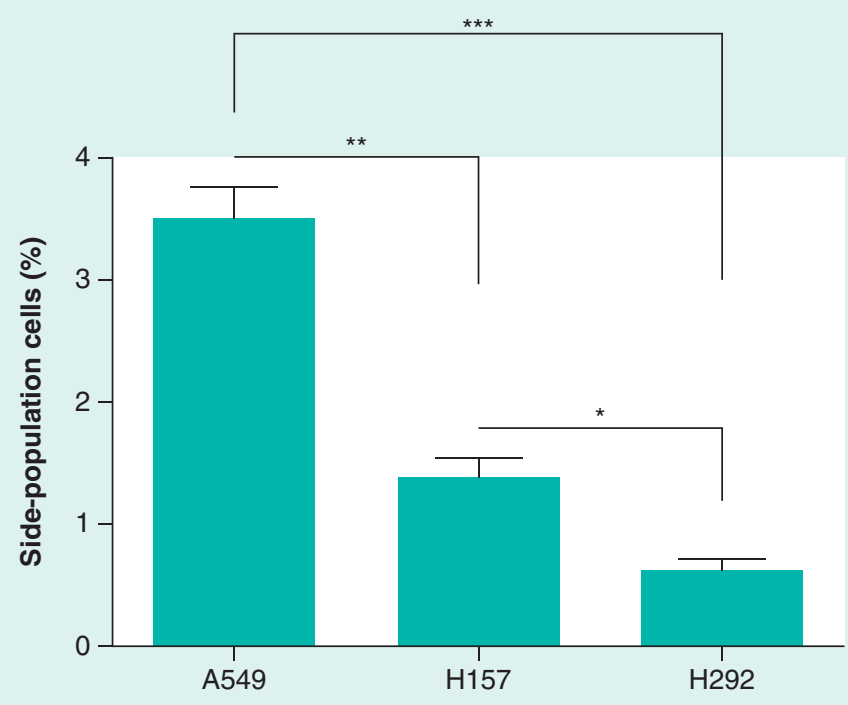

Figure 2. Claudin-3 expression is associated with cancer stemness in nonsquamous non-small-cell lung carcinoma (cont.). (A) CLDN-3 expression profile from Gene Expression Omnibus database (GDS5410) in which CSCs were isolated from H460 and allowed to redifferentiate under adherent conditions in serum-containing media for up to $24 \mathrm{~h}$. (B) Sorting of SP from H460 cells using BD Aria. Verapamil was used as the negative control for SP analysis. (C) Expression of claudin-3 as well as CSCs markers in H460 and H460 SP. (D) Detection of claudin-3 protein levels between parental H460 cells and H460 tumorspheres. (E) Detection of claudin-3 protein levels among nonsquamous NSCLC cell lines including A549, H157 and H292. (F) Detection of SP percentage among various non-squamous NSCLC cell lines including A549, H157 and H292. Data of SP analysis from triplicates are presented as the mean $\pm \mathrm{standard}$ deviation; ${ }^{*} \mathrm{p}$ $<0.05 ; * * p<0.01 ; * * * p<0.001$.

CSC: Cancer stem-like cell; NSCLC: Non-small-cell lung carcinoma; SP: Side-population. 


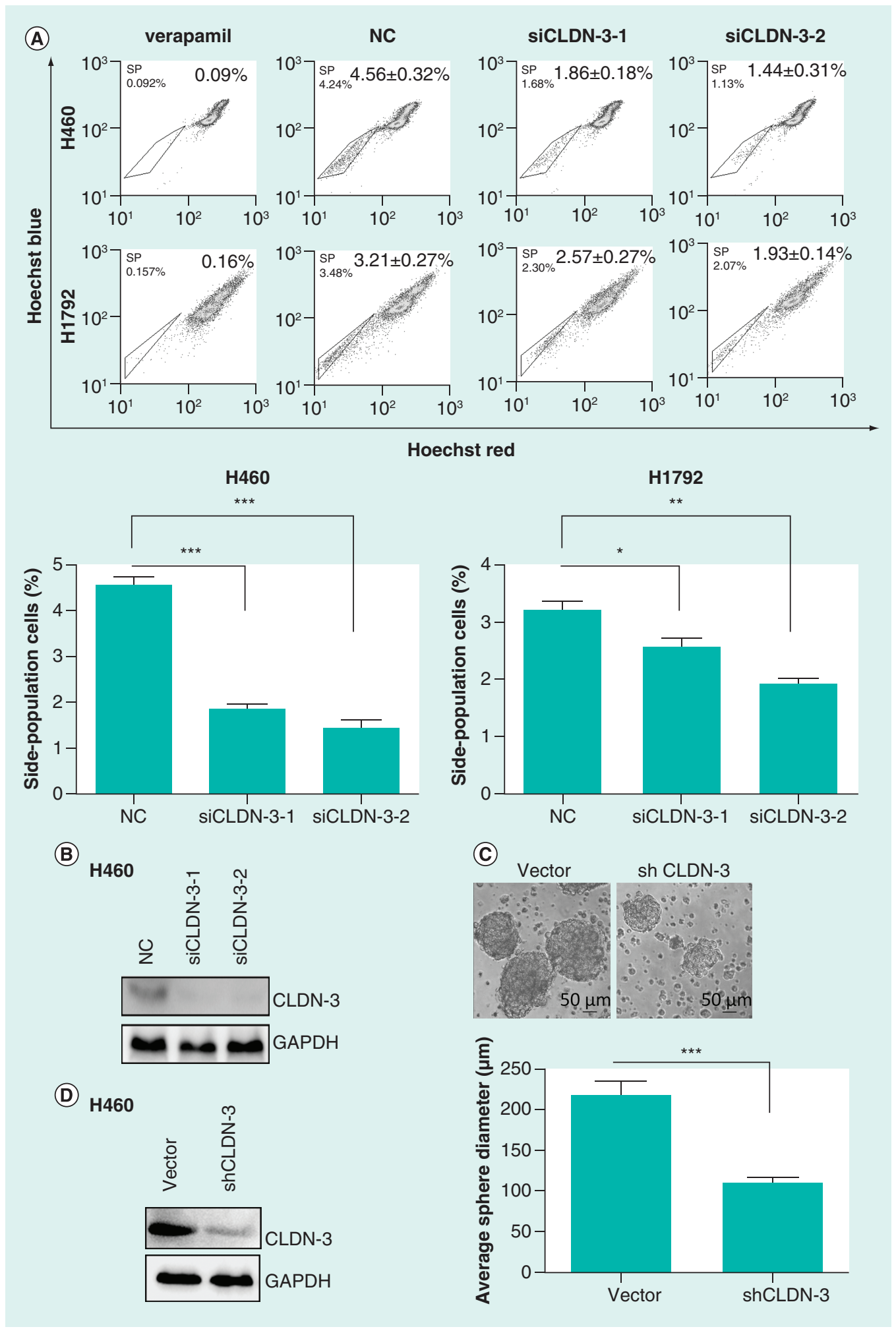

Figure 3. Claudin-3 is a positive regulator of cancer stemness in nonsquamous non-small-cell lung carcinoma. (A) Effect of siRNA-mediated claudin-3 knockdown on the percentage of side-population in $\mathrm{H} 460$ and H1792. (B) The efficiency of claudin-3 knockdown using semiquantitative RT PCR analysis. (C) Effect of claudin-3 knockdown on the capacities of tumorsphere formation ( $n=8$ per group). (D) The efficiency of claudin-3 knockdown using shclaudin-3 lentivirus particles. (E) Effect of claudin-3 knockdown on the rates of tumorsphere formation. (F) Effect of claudin-3 knockdown on the rates of tumor formation. Data of SP analysis from triplicates are presented as the mean \pm standard deviation, ${ }^{*} \mathrm{p}<0.05 ; * * \mathrm{p}<0.01 ; * * * \mathrm{p}<0.001$. The $\mathrm{p}$-value for $\mathrm{E}$ or $\mathrm{F}$ was calculated using the online Extreme Limiting Dillution Analysis (ELDA) online program at http://bioinf.wehi.edu.au/software/elda/.RT PCR: Reverse transcription PCR; SP: Side-population. 
(E)

\begin{tabular}{|l|l|l|l|l|l|l|l|l|}
\hline \multirow{3}{*}{ H460 } & \multicolumn{3}{|l|}{ Sphere-containing wells/total wells } & \multirow{2}{*}{ TCF(95\% Cl) } & TCF estimate & Fold & $p$-value \\
\cline { 2 - 5 } & Cells per well & & & & \\
\cline { 2 - 7 } & $1 \times 10^{3}$ & $1 \times 10^{2}$ & $1 \times 10^{1}$ & $1 \times 10^{0}$ & & & & \\
\hline Vector & $96 / 96$ & $74 / 96$ & $37 / 96$ & $3 / 96$ & $1 / 61.5-1 / 39.8$ & $1 / 49.5$ & 1.0 & $p<0.001$ \\
\hline shCLDN-3 & $88 / 96$ & $53 / 96$ & $13 / 96$ & $0 / 96$ & $1 / 287.5-1 / 174.1$ & $1 / 223.7$ & 0.2 & \\
\hline
\end{tabular}

(F)

\begin{tabular}{|l|l|l|l|l|l|l|l|l|}
\hline \multirow{3}{*}{ H460 } & \multicolumn{4}{|l|}{ Numbers of tumors/total injections } & \multirow{2}{*}{ TCF $(95 \%$ Cl $)$} & $\begin{array}{l}\text { TCF } \\
\text { estimate }\end{array}$ & Fold & $p$-value \\
\cline { 2 - 5 } & \multicolumn{2}{|l|}{ Cells per injection } & & & \\
\cline { 2 - 7 } & $1 \times 10^{6}$ & $1 \times 10^{5}$ & $1 \times 10^{4}$ & $1 \times 10^{3}$ & & $1 / 0$ & $\mathrm{p}<0.05$ \\
\hline Vector & $8 / 8$ & $6 / 8$ & $4 / 8$ & $1 / 8$ & $1 / 133654-1 / 28719$ & $1 / 61955$ & 1.0 \\
\hline shCLDN-3 & $6 / 8$ & $3 / 8$ & $1 / 8$ & $0 / 8$ & $1 / 666710-1 / 130558$ & $1 / 295033$ & 0.2 & \\
\hline
\end{tabular}

Figure 3. Claudin-3 is a positive regulator of cancer stemness in nonsquamous non-small-cell lung carcinoma (cont.). (A) Effect of siRNA-mediated claudin-3 knockdown on the percentage of side-population in H460 and H1792. (B) The efficiency of claudin-3 knockdown using semiquantitative RT PCR analysis. (C) Effect of claudin-3 knockdown on the capacities of tumorsphere formation ( $\mathrm{n}=8$ per group). (D) The efficiency of claudin-3 knockdown using shclaudin-3 lentivirus particles. (E) Effect of claudin-3 knockdown on the rates of tumorsphere formation. (F) Effect of claudin-3 knockdown on the rates of tumor formation. Data of SP analysis from triplicates are presented as the mean \pm standard deviation, ${ }^{*} p<0.05 ; * x<0.01 ; * * * p<0.001$. The $p$-value for E or F was calculated using the online Extreme Limiting Dillution Analysis (ELDA) online program at http://bioinf.wehi.edu.au/software/elda/.RT PCR: Reverse transcription PCR; SP: Side-population.

downregulated the transcription and protein levels of claudin-3 (Figures $6 \mathrm{C} \& \mathrm{D}$ ). On the contrary, claudin-3 depletion has little effect on the level of ER- $\alpha$ mRNA (Figure 6E). To further investigate the relationship between ER- $\alpha$ and claudin- 3 in regulating stemness in nonsquamous NSCLC, SP assay was performed and the results showed that ER- $\alpha$ knockdown significantly led to the downregulation of the frequency of SP cells. However, ER- $\alpha$ silencing almost failed to decrease the percentage of SP in claudin-3-depleted H460 cells (Figure 6F). Therefore, claudin-3 could function as the downstream of ER- $\alpha$ in mediating cancer stemness in nonsquamous NSCLC.

\section{Discussion}

Tight junctions are the most apical component of the junctional complex in epithelial or endothelial cells and form a barrier to paracellular movement of substances separating the apical and basolateral fluid compartments on opposite sides of the epithelial cell layer. As the pivotal element in the maintenance of tight junctions, claudin proteins are abnormally expressed in various malignancies [33,34]. Recent studies have shown that claudin proteins play an important role in epithelial to mesenchymal transition (EMT), the formation of CSCs or tumor-initiating cells and chemoresistance $[16,20,35,36]$, suggesting that claudins are promising targets for the treatment of chemoresistant and recurrent tumors. In this work, the role of claudin-3 in mediating cancer stemness in nonsquamous NSCLC has been preliminarily elaborated. As claudin-3 contains two extracellular domains, which are accessible as target sites, targeting claudin-3 will help provide promising strategies to overcome stemness in treatment of nonsquamous NSCLC. As one kind of toxin produced by Clostridium species, which are the major causes of food poisoning/gastrointestinal illnesses, Clostridium perfringens enterotoxin can bind with Claudin-3 and induce rapid cytolysis [37]. Currently, CPE-based therapy has been widely used in targeted therapy against malignancies in which claudin-3, the receptor of CPE, is highly expressed [38,39]. Considering that claudin-3 has been identified as a positive regulator of stemness in nonsquamous NSCLC, CPE-based therapy could represent a highly effective strategy to specifically target chemotherapy-resistant claudin-3-positive CSCs for therapeutic purposes.

Here, we have identified several small molecules including estradiol, WA and fulvestrant which could all downregulate claudin-3 level and suppress cancer stemness in nonsquamous NSCLC. Among these molecules, WA has shown the strongest inhibitory effect on claudin-3 expression and cancer stemness. As a steroidal lactone occurring in Withania somnifera, WA exhibited cytotoxicity in a variety of tumor cell lines and in animal cancer models without any noticeable systemic toxicity [40,41]. However, the mechanism of its action is currently under 

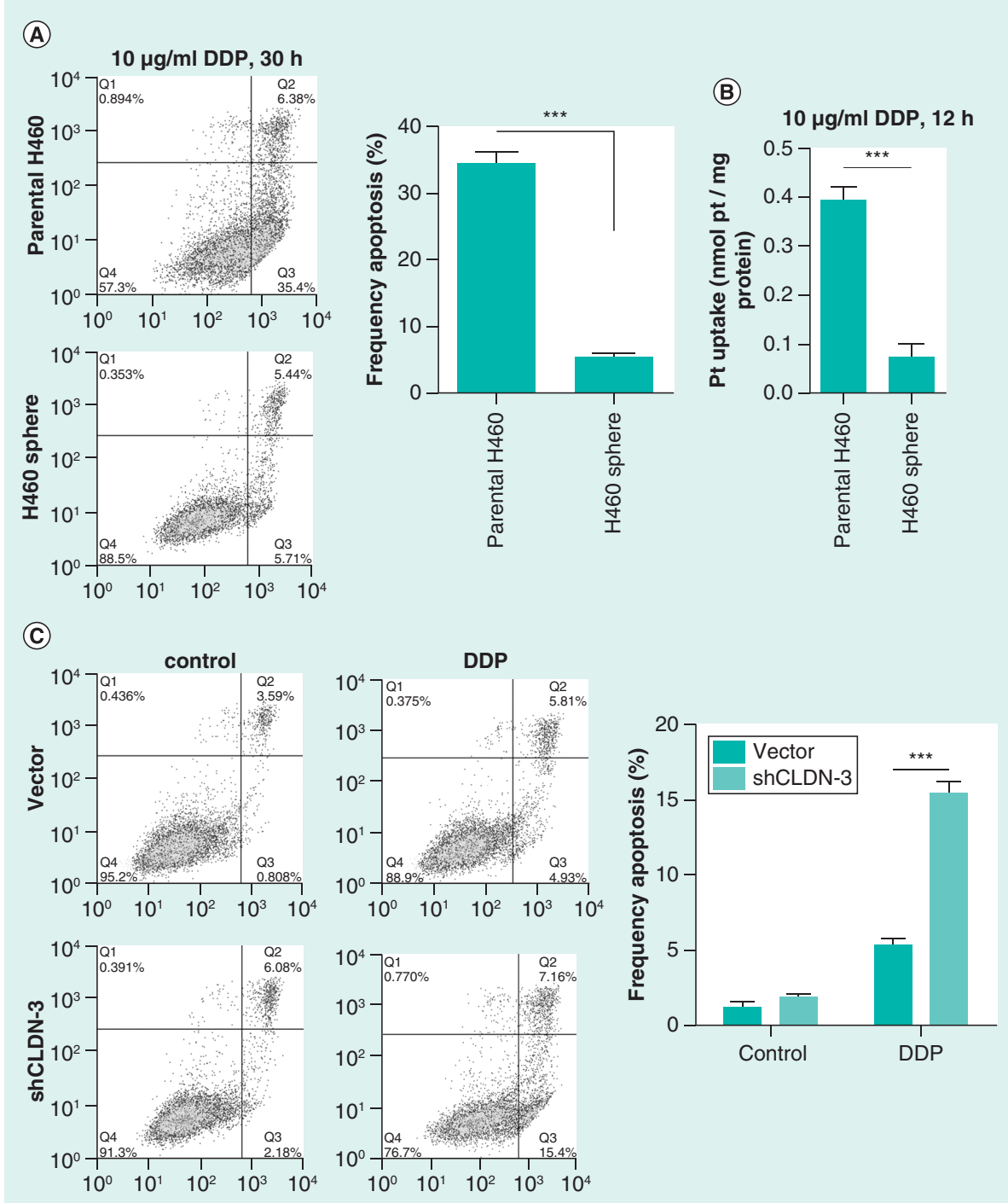

(D)

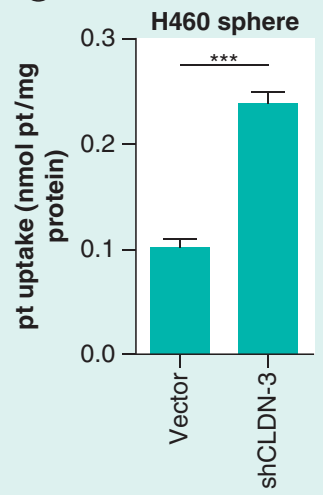

(E)

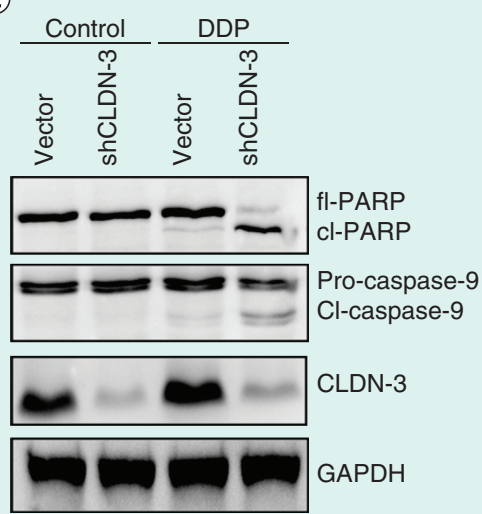

Figure 4. Claudin-3 is a positive regulator of cancer stem-like cells-mediated cisplatin resistance in nonsquamous non-small-cell lung carcinoma. (A) Comparison of cisplatin sensitivities between parental H460 cells and H460 tumorspheres. (B) Comparison of cisplatin uptake between parental $\mathrm{H} 460$ cells and H460 tumorspheres. (C) Effect of claudin-3 knockdown on cisplatin resistance in H460 tumorspheres. (D) Effect of claudin-3 knockdown on cisplatin uptake in $\mathrm{H} 460$ tumorspheres. (E) Effect of claudin-3 knockdown on cisplatin-induced apoptosis-related proteins including caspase-9 and its downstream poly ADP-ribose polymerase (PARP) in H460 tumorspheres. Data of apoptosis and ICP MS analysis from triplicates are presented as the mean \pm standard deviation, $* * * p<0.001$. 


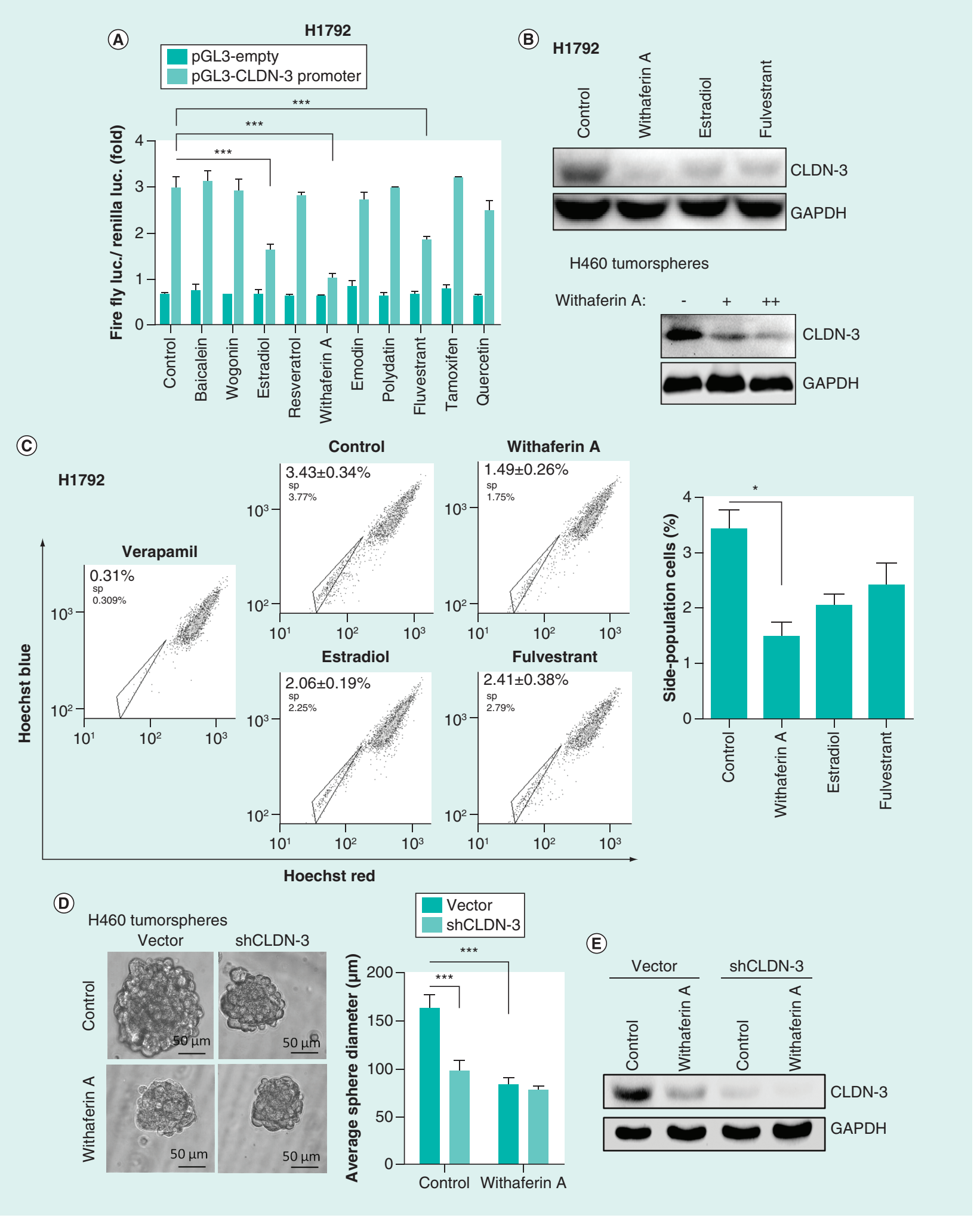

Figure 5. Claudin-3-based drug screening to suppress cancer stemness in nonsquamous non-small-cell lung carcinoma. (A) Effect on small molecules on CLDN-3 transcription. (B) Effect of estradiol, WA and fulvestrant on the claudin-3 protein level. (C) Effect of estradiol, WA and fulvestrant on the percentage of SP in H1792. (D) Effect of WA and claudin-3 knockdown on the capacities of tumorsphere formation ( $n=8$ per group). (E) Effect of WA and claudin-3 knockdown on the claudin-3 protein level. (F) Effect of WA and claudin-3 knockdown on the tumorsphere formation rate. (G) Effect of WA and claudin-3 knockdown on the cisplatin sensitivity in H460 tumorspheres. Data of apoptosis, luciferase reporter analysis and SP analysis from triplicates are presented as the mean \pm standard deviation; ${ }^{*}<0.05 ; * * * p<0.001$. The $p$-value for (F) was calculated using the online ELDA software. DDP: SP: Side-population; TCF: WA: Withaferin A. 
(F)

\begin{tabular}{|c|c|c|c|c|c|c|c|c|}
\hline \multirow[t]{3}{*}{$\mathrm{H} 460$} & \multicolumn{4}{|c|}{ Sphere-containing wells/total wells } & \multirow[t]{3}{*}{ TCF $(95 \% \mathrm{Cl})$} & \multirow[t]{3}{*}{ TCF estimate } & \multirow[t]{3}{*}{ fold } & \multirow[t]{3}{*}{$p$-value } \\
\hline & \multicolumn{4}{|c|}{ Cells per well } & & & & \\
\hline & $1 \times 10^{3}$ & $1 \times 10^{2}$ & $1 \times 10^{1}$ & $1 \times 10^{0}$ & & & & \\
\hline Vector (control) & $48 / 48$ & $40 / 48$ & $14 / 48$ & $1 / 48$ & $1 / 65-1 / 35.1$ & $1 / 47.7$ & 1.0 & \multirow[t]{2}{*}{$p<0.001$} \\
\hline Vector (WA) & $41 / 48$ & $19 / 48$ & $6 / 48$ & $0 / 48$ & $1 / 485-1 / 255.2$ & $1 / 351.7$ & 0.1 & \\
\hline shCLDN-3 (control) & $45 / 48$ & $23 / 48$ & $4 / 48$ & $0 / 48$ & $1 / 337-1 / 167.5$ & $1 / 237.7$ & 0.2 & \multirow[t]{2}{*}{ N.S. $(p>0.05)$} \\
\hline shCLDN-3 (WA) & $42 / 48$ & $17 / 48$ & $4 / 48$ & $0 / 48$ & $1 / 498-1 / 263.3$ & $1 / 362.2$ & 0.1 & \\
\hline
\end{tabular}

(G)

DDP

DDP + withaferin A
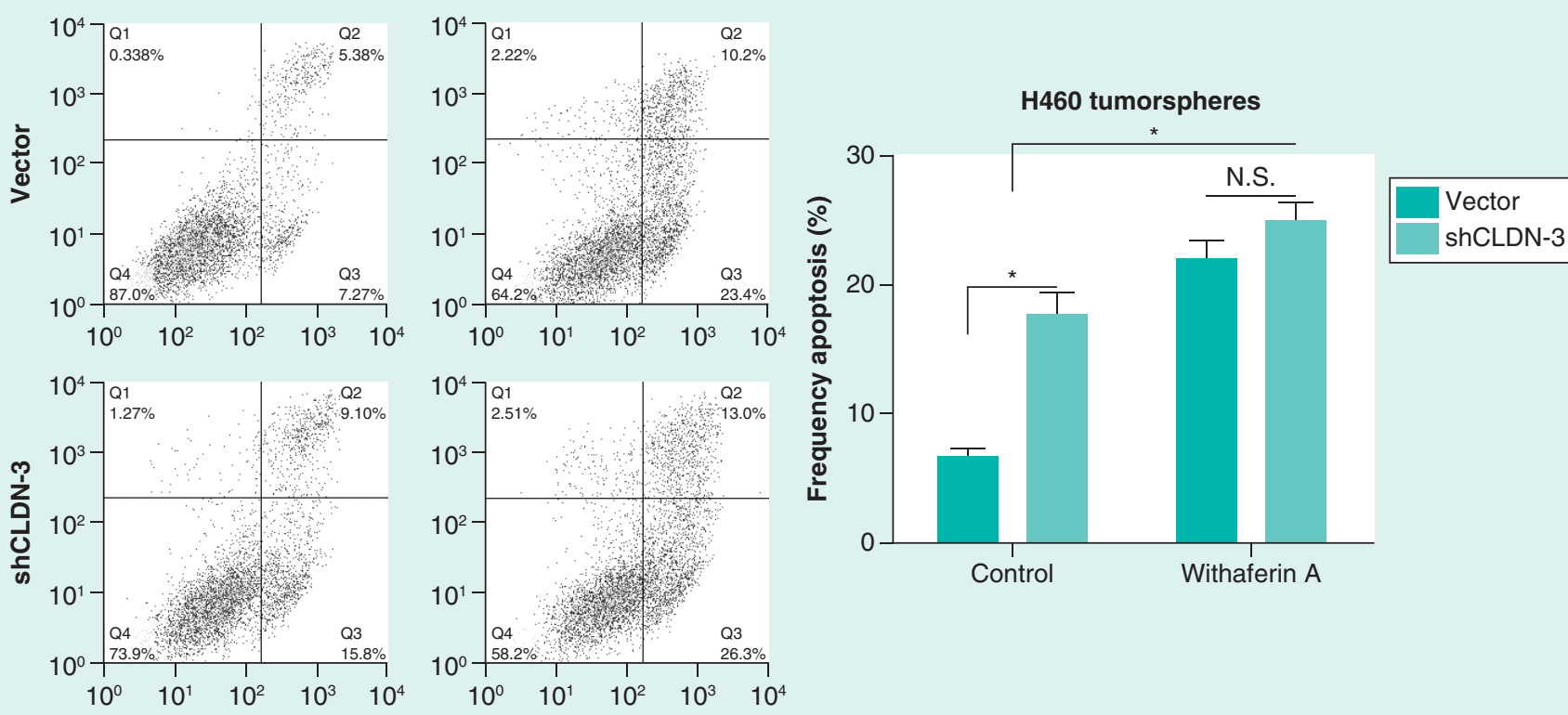

Figure 5. Claudin-3-based drug screening to suppress cancer stemness in nonsquamous non-small-cell lung carcinoma (cont.). (A) Effect on small molecules on CLDN-3 transcription. (B) Effect of estradiol, WA and fulvestrant on the claudin-3 protein level. (C) Effect of estradiol, WA and fulvestrant on the percentage of SP in H1792. (D) Effect of WA and claudin-3 knockdown on the capacities of tumorsphere formation ( $n=8$ per group). (E) Effect of WA and claudin-3 knockdown on the claudin-3 protein level. (F) Effect of WA and claudin-3 knockdown on the tumorsphere formation rate. (G) Effect of WA and claudin-3 knockdown on the cisplatin sensitivity in H460 tumorspheres. Data of apoptosis, luciferase reporter analysis and SP analysis from triplicates are presented as the mean \pm standard deviation; $* p<0.05 ; * * * p<0.001$. The $p$-value for $(F)$ was calculated using the online ELDA software.

DDP: SP: Side-population; TCF: WA: Withaferin A.

extensive investigation. It has been demonstrated that WA has the ability to alter numerous cancer-associated growth factor receptors, kinases and transcription factors and the ability to inhibit nuclear factor- $\kappa \mathrm{B}$ activation and angiogenesis [29,42-44]. In this work, WA was found to suppress cancer stemness by repressing claudin-3 expression. Hence, this novel function of WA could provide a promising way of sensitizing CSCs-mediated chemoresistance in nonsquamous NSCLC.

Another important strength of this study is that the relationship between claudin-3 and ER- $\alpha$ in nonsquamous NSCLC has been uncovered. Estrogen is vital for normal postpubertal mammary development and long-term exposure to estrogen is proven to be associated with an increased risk of breast cancer [45]. However, the research area of estrogen signaling is not restricted in breast cancer. Considerable data showed that malignancies including NSCLC, CRC and all associated with estrogen signaling [46-48]. A focus on ERs as potential indicators of prognosis has played a significant role in the diagnosis and/or treatment of NSCLC [49]. In this study, targeting ER- $\alpha$ by small molecules or siRNAs was found to downregulate the percentage of SP with decreased claudin-3 levels, suggesting that ER- $\alpha$ could also be a contributing factor to cancer stemness in nonsquamous NSCLC similarly to the case in breast cancer, by downregulating claudin-3 in regulating cancer stemness. This speculation can also be confirmed by the claudin-3 expression profile from the Gene Expression Omnibus database (GDS4061) in which claudin-3 

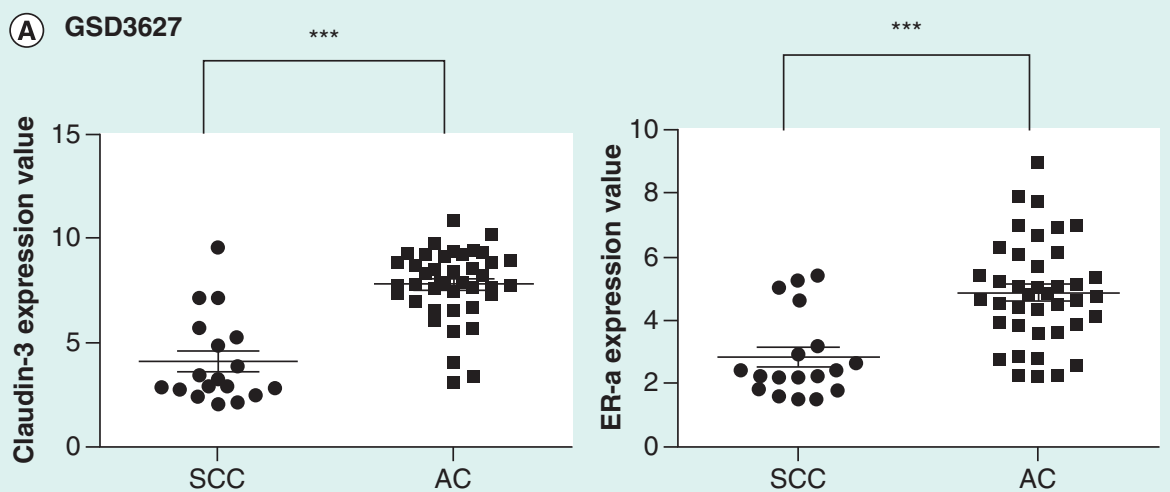

(B) GDS4061

Title Estrogen receptor alpha-silenced MCF7 breast cancer cells

Organism Homo sapiens

\section{Claudin-3}

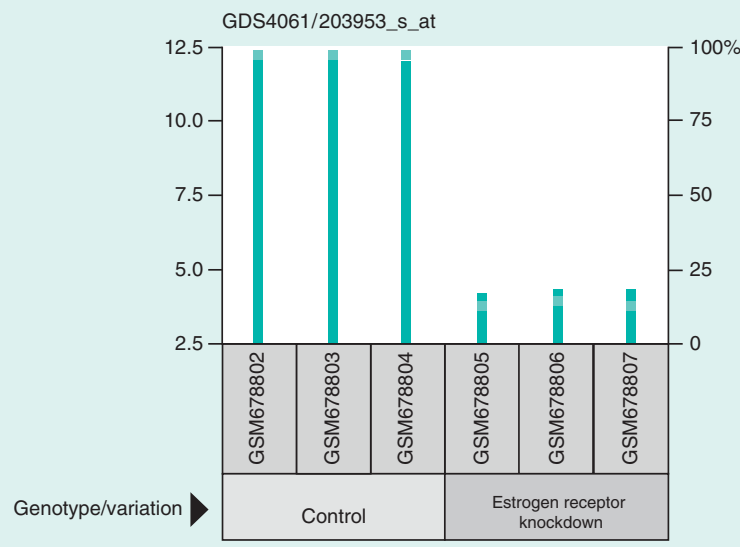

\begin{tabular}{|l|}
\hline Transformed count \\
Percentile rank within the sample
\end{tabular}

CD24

GDS4061/216379 X at

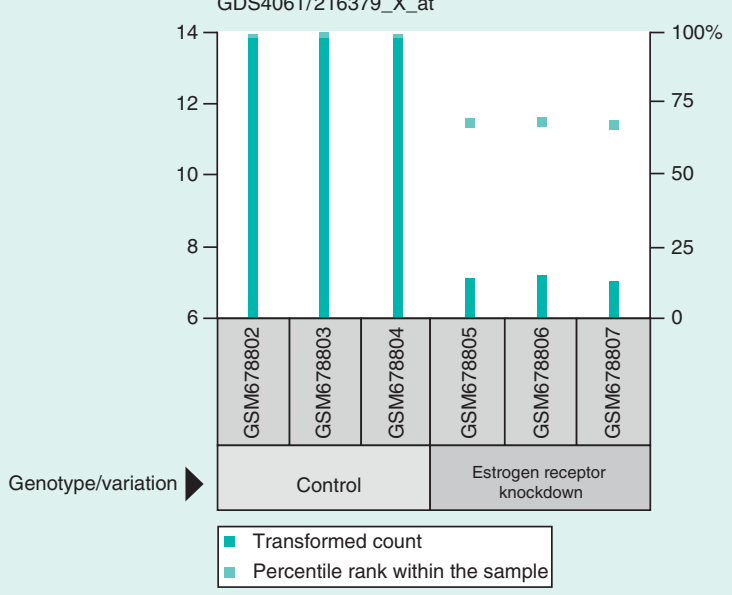

CD133

GDS4061/204304_s_at

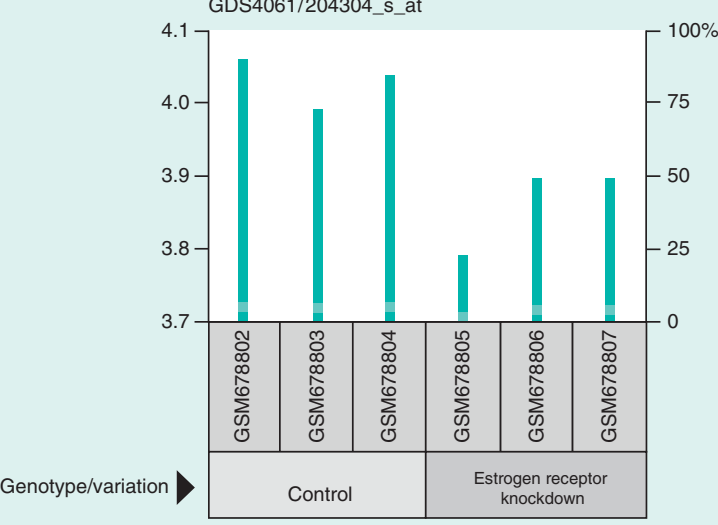

\begin{tabular}{|l|l|} 
Transformed count \\
Percentile rank within the sample
\end{tabular}

ABCG2

GDS4061/209735_at

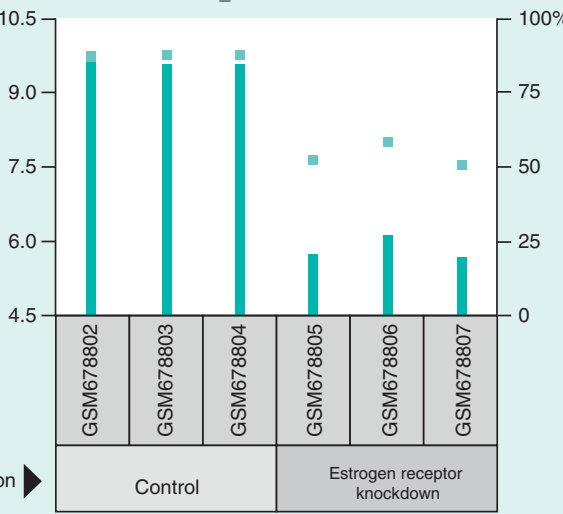

- Transformed count

Percentile rank within the sample

Figure 6. Claudin-3 functions downstream of estrogen receptor- $\alpha$ in nonsquamous non-small-cell lung carcinoma. (A) Expression analysis of ER- $\alpha$ and claudin-3 in AC and SCC samples from GEO database (GDS3627). (B) Effect of ER- $\alpha$ knockdown on expression of claudin-3 as well as CSCs markers including CD133, CD24, ABCG2 in the breast cancer cell line MCF7. Data were obtained from GEO database (GDS4061). (C) Effect of ER- $\alpha$ knockdown on the claudin-3 protein level in H1792. (D) Effect of ER- $\alpha$ knockdown on claudin-3 transcription in H1792. (E) Effect of ER- $\alpha$ and claudin-3 double knockdown on ER- $\alpha$ or claudin-3 expression in H460. (F) Effect of ER- $\alpha$ and claudin-3 double knockdown on the percentage of SP in H460. Data from triplicates are presented as the mean \pm standard deviation; $* * p<0.01 ; * * * p<0.001$.

CSC: Cancer stem-like cell; ER- $\alpha$ : Estrogen receptor- $\alpha$; GEO: Gene Expression Omnibus; SCC: Squamous cell lung carcinoma; SP: Side-population. 
(C)

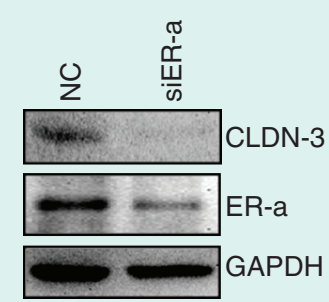

(D)

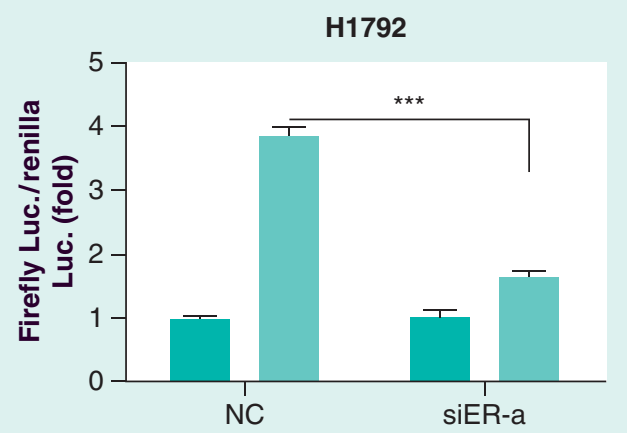

(E)

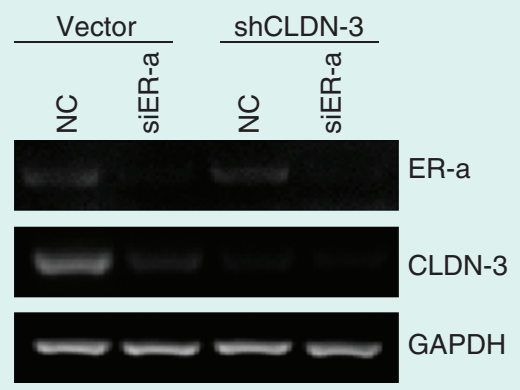

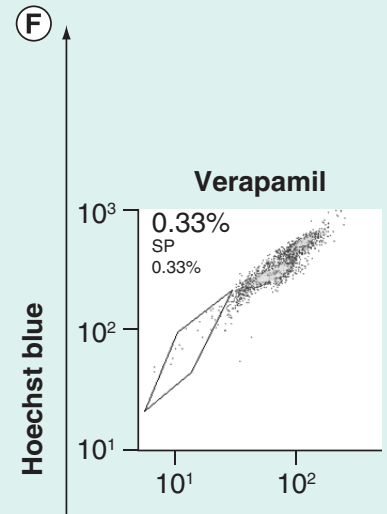
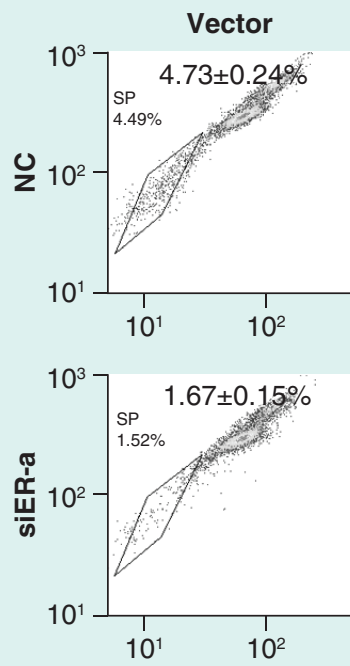

ShCDLN-3
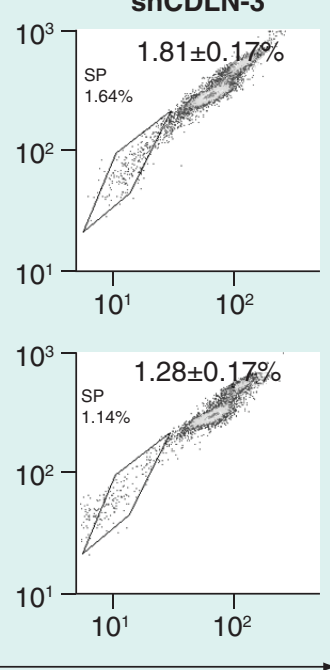

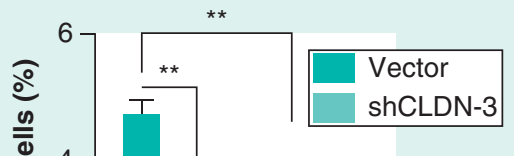

Hoechst red

Figure 6. Claudin-3 functions downstream of estrogen receptor- $\alpha$ in nonsquamous non-small-cell lung carcinoma (cont.). (A) Expression analysis of ER- $\alpha$ and claudin-3 in AC and SCC samples from GEO database (GDS3627). (B) Effect of ER- $\alpha$ knockdown on expression of claudin-3 as well as CSCs markers including CD133, CD24, ABCG2 in the breast cancer cell line MCF7. Data were obtained from GEO database (GDS4061). (C) Effect of ER- $\alpha$ knockdown on the claudin-3 protein level in H1792. (D) Effect of ER- $\alpha$ knockdown on claudin-3 transcription in H1792. (E) Effect of ER- $\alpha$ and claudin-3 double knockdown on ER- $\alpha$ or claudin-3 expression in H460. (F) Effect of ER- $\alpha$ and claudin-3 double knockdown on the percentage of SP in H460. Data from triplicates are presented as the mean \pm standard deviation; ${ }^{* *} \mathrm{p}<0.01 ; * * * \mathrm{p}<0.001$.

CSC: Cancer stem-like cell; ER- $\alpha$ : Estrogen receptor- $\alpha$; GEO: Gene Expression Omnibus; SCC: Squamous cell lung carcinoma; SP: Side-population.

was markedly downregulated in ER- $\alpha$ knockdown MCF7 cells. Thus, claudin-3 could function downstream of ER- $\alpha$ and positive regulates cancer stemness in nonsquamous NSCLC and strategies to suppress claudin- 3 using ER- $\alpha$ inhibitors may be potential anti-CSCs therapies in the treatment of nonsquamous NSCLC.

\section{Conclusion}

This benchmarking exercise characterized the role of claudin-3 as a positive regulator of cancer stemness in nonsquamous NSCLC and confirmed the relationship between claudin-3 and its upstream ER- $\alpha$. Moreover, claudin-3-targeted therapies using small molecules including ER- $\alpha$ inhibitors could contribute to specifically target CSCs for nonsquamous NSCLC therapy. 


\section{Future perspective}

Although the role of claudin-3 in CSCs has been defined in nonsquamous NSCLC, the mechanisms by which claudin-3 regulates cancer stemness have yet to be explored in depth in further research. Whether targeting claudin3 suppresses tumor relapse after chemotherapy or not still remains to be investigated. Moreover, establishment of a systemic high-throughput drug screening system based on this target could be a promising strategy against nonsquamous NSCLC in future.

\section{Summary points}

- Claudin-3 is overexpressed in nonsquamous non-small-cell lung carcinoma (NSCLC).

- Claudin-3 expression is associated with cancer stemness in nonsquamous NSCLC.

- Claudin-3 is a positive regulator of cancer stemness in nonsquamous NSCLC.

- Claudin-3 is a positive regulator of cancer stem-like cells-mediated cisplatin resistance in nonsquamous NSCLC.

- Withaferin A was found to suppress cancer stemness by repressing claudin-3 expression.

- Claudin-3 level positively correlated with estrogen receptor- $\alpha$ (ER- $\alpha)$.

- Transcription of claudin-3 requires ER- $\alpha$.

- Claudin-3 functions as one downstream target of ER- $\alpha$.

\section{Acknowledgments}

We also would like to acknowledge the interactive web server for analyzing the RNA sequencing expression data from the TCGA and the GTEx projects developed by Z Tang, C Li and B Kang of Zhang Lab, Peking University [27].

Financial \& competing interests disclosure

This project was sponsored by Natural Science Fund of China (grant numbers 31071250, 81673462, 81473293, 91540119 and J1103521), the Fundamental Research Funds for the Central Universities. Six talent peaks project in Jiangsu Province to W Yin (YY012). The funder had no role in study design, data collection and analysis, decision to publish or preparation of the manuscript. The authors have no other relevant affiliations or financial involvement with any organization or entity with a financial interest in or financial conflict with the subject matter or materials discussed in the manuscript apart from those disclosed.

No writing assistance was utilized in the production of this manuscript.

\section{Open access}

This work is licensed under the Attribution-NonCommercial-NoDerivatives 4.0 Unported License. To view a copy of this license, visit http://creativecommons.org/licenses/by-nc-nd/4.0/

\section{References}

Papers of special note have been highlighted as: $\bullet$ of interest; $\bullet \bullet$ of considerable interest

1. Siegel RL, Miller KD, Jemal A. Cancer statistics, 2016. CA Cancer J. Clin. 66(1), 7-30 (2016).

2. Skrzypski M, Dziadziuszko R, Jassem E et al. Main histologic types of non-small-cell lung cancer differ in expression of prognosis-related genes. Clin. Lung Cancer. 14(6), 666-673 (2013).

3. Weiss MC, Leukam MJ, Villaflor VM. Molecular landscape of non-squamous, non-small-cell carcinoma of the lung. Transl. Cancer Res. 4(4), 423-434 (2015).

4. Li TC, Li CI, Tseng CH et al. Quality of life predicts survival in patients with non-small-cell lung cancer. BMC Public Health 12, 790 (2012).

5. Abdullah LN, Chow KH. Mechanisms of chemoresistance in cancer stem cells. Clin. Transl. Med. 2, 3 (2013).

6. Templeton AK, Miyamoto S, Babu A, Munshi A, Ramesh R. Cancer stem cells: progress and challenges in lung cancer. Stem Cell Investig. 1(9), 1-18 (2014).

7. Morrison RD, Schleicher S, Sun Y et al. Targeting the mechanisms of resistance to chemotherapy and radiotherapy with the cancer stem cell hypothesis. J. Oncol. 2011(12), 941876 (2011).

8. Krause G, Winkler L, Mueller SL et al. Structure and function of claudins. Biochim. Biophys. Acta 1778(3), 631-645 (2008).

9. Morita K, Furuse M, Fujimoto K, Tsukita S. Claudin multigene family encoding four-transmembrane domain protein components of tight junction strands. Proc. Natl Acad. Sci. USA 96(2), 511-516 (1999).

10. Singh AB, Sharma A, Dhawan P. Claudin family of proteins and cancer: an overview. J. Oncol. 2010, 541957 (2010). 
11. Hoggard J, Fan J, Lu Z, Lu Q, Sutton L, Chen YH. Claudin-7 increases chemosensitivity to cisplatin through the upregulation of caspase pathway in human NCI-H522 lung cancer cells. Cancer Sci. 104(5), 611-618 (2013).

- Claudin-7 could be a suppressor in lung cancer.

12. Lu Z. Functions of claudin-7 in human lung cancer. Doctoral Dissertation, East Carolina University, NC USA. (2012). http://hdl.handle.net/10342/4026

13. Chae MC, Park CK, Keum DY, Hwang I, Kwon KY, Jang BC. Prognostic significance of claudin-4 in completely resected adenocarcinoma of the lung. Korean J. Thorac. Cardiovasc. Surg. 47(3), 262-268 (2014).

14. Chaouche-Mazouni S, Scherpereel A, Zaamoum R et al. Claudin-3, -4, and -15 expression in solid tumors of lung adenocarcinoma versus malignant pleural mesothelioma. Ann. Diagn. Pathol. 19(4), 193-197 (2015).

- Members of the claudin family could expressed and function differently.,

15. Huo Q, Kinugasa T, Wang L et al. Claudin-1 protein is a major factor involved in the tumorigenesis of colorectal cancer. Anticancer Res. 29(3), 851-857 (2009).

- The role of claudin-1 in mediating cancer stemness in colorectal cancer is interesting.

16. Myal Y, Leygue E, Blanchard AA. Claudin 1 in breast tumorigenesis: revelation of a possible novel "claudin high" subset of breast cancers. BioMed Res. Int. 2010, 1-10 (2010).

- The role of claudin-1 in mediating tumorigenesis in breast cancer stemness is interesting.

17. Swisshelm K, Macek R, Kubbies M. Role of claudins in tumorigenesis. Adv. Drug Deliv. Rev. 57(6), 919-928 (2005).

18. Pan X, Li X, Che Y, Li H et al. Overexpression of claudin-4 may be involved in endometrial tumorigenesis. Oncol. Lett. 5(4), 1422-1426 (2013).

- The role of claudin-1 in mediating endometrial tumorigenesis is interesting.

19. Ahmad R, Kumar B, Chen Z et al. Loss of claudin-3 expression induces IL6/gp130/Stat3 signaling to promote colon cancer malignancy by hyperactivating Wnt/ $\beta$-catenin signaling. Oncogene 36(47), 6592-6604 (2017).

-. The role of claudin-3 in colon cancer and lung adenocarcinoma could be different.

20. Jiang L, Yang YD, Fu L et al. CLDN3 inhibits cancer aggressiveness via Wnt-EMT signaling and is a potential prognostic biomarker for hepatocellular carcinoma. Oncotarget 5(17), 7663-7676 (2014).

-. The role of claudin-3 in hepatocellular carcinoma and lung adenocarcinoma could be different.

21. Huang Y, Bao Y, Peng W et al. Claudin-3 gene silencing with siRNA suppresses ovarian tumor growth and metastasis. Proc. Natl Acad. Sci USA106(9), 3426-3430 (2009).

22. Todd MC, Petty HM, King JM et al. Overexpression and delocalization of claudin-3 protein in MCF-7 and MDA-MB-415 breast cancer cell lines. Oncol. Lett. 10(1), 156-162 (2015).

23. Dhawan P, Ahmad R, Chaturvedi R et al. Claudin-2 expression increases tumorigenicity of colon cancer cells: role of epidermal growth factor receptor activation. Oncogene30(29), 3234-3247 (2011).

24. Kinugasa T, Yoshito A, Yoshida T et al. Increased expression of claudin-1 contributes to tumorigenesis in ulcerative colitis-associated colorectal cancer. Anticancer Res. 30(8), 3181-3186 (2011).

25. Shi Y, Fu X, Hua Y et al. The side population in human lung cancer cell line NCI-H460 is enriched in stem-like cancer cells. PLoS ONE 7(3), e33358 (2012).

26. Walter + Eliza Hall Bioinformatics. ELDA: extreme limiting dilution analysis. http://bioinf.wehi.edu.au/sof tware/elda/

27. Tang Z, Li C, Kang B et al. GEPIA: a web server for cancer and normal gene expression profiling and interactive analyses. Nucleic Acids Res. 45(W1), W98-W102 (2017).

28. National Center for Biotechnology Information. Gene expression omnibus. www.ncbi.nlm.nih.gov/geo/query/acc.cgi

29. Lopez-Ayllon BD, Moncho-Amor V, Abarrategi A et al. Cancer stem cells and cisplatin-resistant cells isolated from non-small-lung cancer cell lines constitute related cell populations. Cancer Med. 3(5), 1099-1111 (2015).

30. Hahm E, Lee J, Huang Y et al. Withaferin a suppresses estrogen receptor- $\alpha$ expression in human breast cancer cells. Mol. Carcinog. 50(8), 614-624 (2011).

31. Hall JM, Couse JF, Korach KS. The multifaceted mechanisms of estradiol and estrogen receptor signaling. J. Biol. Chem. 276(40), 36869-36872 (2001).

32. Jiang D, Huang $\mathrm{Y}$, Han $\mathrm{N}$ et al. Fulvestrant, a selective estrogen receptor down-regulator, sensitizes estrogen receptor negative breast tumors to chemotherapy. Cancer Lett. 346(2), 292-299 (2014).

33. Kwon MJ. Emerging roles of claudins in human cancer. Int. J. Mol. Sci. 14(9), 18148-18180 (2013).

34. Morin PJ. Claudin proteins in human cancer: promising new targets for diagnosis and therapy. Cancer Res. 65(21), 9603-9606 (2005).

35. Turksen K. Claudins and cancer stem cells. Stem Cell Rev. 7(4), 797-798 (2011).

36. Hicks DA, Galimanis CE, Webb PG et al. Claudin-4 activity in ovarian tumor cell apoptosis resistance and migration. BMC Cancer 16(1), 788 (2016). 
- The function of claudin- 4 in regulating apoptosis resistance and migration in ovarian cancer is interesting.

37. Miyakawa MEF, Creydt VP, Uzal FA, Clane BA, Ibarra C. Clostridium perfringens. Enterotoxin damages the human intestine in vitro. Infect. Immun. 73(12), 8407-8410 (2005).

38. Romanov V, Whyard TC, Waltzer WC, Gabig TG. A claudin 3- and claudin 4-targeted Clostridium perfringens protoxin is selectively cytotoxic to PSA-producing prostate cancer cells. Cancer Lett. 351(2), 260-264 (2014).

39. Walther W, Petkov S, Kuvardina ON, Aumann J et al. Novel Clostridium perfringens enterotoxin suicide gene therapy for selective treatment of claudin-3- and -4-overexpressing tumors. Gene Ther. 19(5), 494-503 (2012).

40. Nishikawa Y, Okuzaki D, Fukushima K et al. Withaferin A induces cell death selectively in androgen-independent prostate cancer cells but not in normal fibroblast cells. PLoS ONE 10(7), e:0134137 (2015).

41. Yang I, Kim L, Shin J, Cho S. Chemotherapeutic effect of withaferin A in human oral cancer cells. J. Cancer Ther. 06(08), 735-742 (2015).

42. Hahm E, Lee J, Singh SV. Role of mitogen-activated protein kinases and Mcl-1 in apoptosis induction by withaferin A in human breast cancer cells. Mol. Carcinog. 53(11), 907-916 (2014).

43. Jackson SS, Oberley CC, Hooper C et al. Withaferin A disrupts ubiquitin-based NEMO reorganization induced by canonical NF- $\mathrm{kB}$ signaling. Exp. Cell Res. 331(1), 58-72 (2015).

44. Mohan R, Hammers HJ, Bargagnamohan P et al. Withaferin A is a potent inhibitor of angiogenesis. Angiogenesis 7(2), 115-122 (2004).

45. Anderson WF, Chatterjee N, Ershler WB, Brawley OW. Estrogen receptor breast cancer phenotypes in the surveillance, epidemiology, and end results database. Breast Cancer Res. Treat. 76(1), 27-36 (2002).

- The result that claudin-3 was downregulated in estrogen receptor- $\alpha$-depleted breast cancer cells was interesting.

46. Luo Z, Wu R, Jiang Y, Qui W, Li W. Overexpression of estrogen receptor beta is a prognostic marker in non-small-cell lung cancer: a meta-analysis. Int. J. Clin. Exp. Med. 8(6), 8686-8697 (2015).

47. Fiorelli G, Picariello L, Martineti V, Tognarini I, Tonelli F, Brandi ML. Estrogen metabolism in human colorectal cancer cells. J. Steroid Biochem. Mol. Biol. 81(3), 281-289 (2002).

48. Seitz G, Pollow K, Hinkeldey K, Schüder G, Wernert N. Immunohistochemical estrogen receptor detection in the pancreas, in pancreatic cancer and other gastrointestinal cancers? Pathol. Res. Pract. 71, 140-143 (1987).

49. Kawai H, Ishii A, Washiya $\mathrm{K}$ et al. Estrogen receptor $\alpha$ and $\beta$ are prognostic factors in non-small-cell lung cancer. Clin. Cancer Res. 11(14), 5084-5089 (2005). 